\title{
NUMERICAL EXPERIMENTS AND NEUTRON STAR FORMATION
}

\author{
W. David Arnett* \\ Enrico Fermi Institute \\ University of Chicago \\ Chicago. IL 60637 \\ USA
}

ABSTRACT. Theory and numerical experiments on neutron star formation are critically reviewed. Several new numerical experiments are summarized, and the importance of advection is discussed.

\section{THEORETICAL CONTEXT}

\section{1. Neutrino Deposition, Diffusion and Shocks}

In this paper we will emphasize the difference between theory (a set of quantitative ideas) and numerical experiment ("computer theory"). Because some of the older theoretical ideas are reappearing in the current models for the explosion mechanism, a brief review is in order. The idea of neutrino deposition is due to Colgate and White (1966). Neutrinos. escaping from a collapsed core. heat the infalling mantle so much that an outgoing shock is formed. which causes the explosion. While at low densities the neutrino flux is simply attenuated, at higher densities the mean free paths are so short that diffusion occurs. Arnett (1966. 1967) found that although the mechanism worked if the neutrino opacities were low enough so that diffusion could carry a lot of energy. higher opacity choked down the neutrino luminosity. trapping the neutrinos in the infalling matter. Mazurek (1975) argued that the neutrinos from electron capture were directly trapped. giving a large excess of neutrinos over antineutrinos, and that these neutrinos would be degenerate. With the discovery of neutral currents and the realization that this gave an increased opacity. it became clear that electron-type neutrinos were indeed strongly trapped and degenerate (Sato 1975: Arnett 1977a). Using these results, and emphasizing that the entropy change and lepton loss in the collapsing core would be small. Bethe. Brown. Applegate and Lattimer (1979) developed a theoretical model in which the core "bounce" at nuclear densities caused an outward shock. This was confirmed by numerical experiments (Hillebrandt 1982). but despite initial euphoria this shock did not prove to be the solution to the supernova problem. Extensive work on the equation of state (cf. review by Lattimer 1981), the electron-capture process (Fuller. Fowler *

A. von Humboldt Awardee, Max-Planck-Institut für Astrophysik, Garching bei München, FRG 
and Newman 1982), and the Initial models (Arnett 1977b. Weaver. Zimmerman and Woosley 1978. Nomoto 1982) restricted the plausible parameter space avallable for numerical experiments, and most (all?) shocks died as they propagated to lower density. The killers were energy losses by (1) photo dissociation of nuclei ( $8 \mathrm{MeV} /$ nucleon) and (2) neutrino escape (Arnett 1983. Lattimer and Burrows 1984). Ironically the role of neutrinos in the numerical experiments changed. from driving to damping the explosion. Considerable effort is being expended to see if this "prompt" mechanism works for any acceptable choice of parameters.

\subsection{Late Mechanisms}

If the initial collapse does not give rise to an explosion directly. what happens then? Wilson (1985) found a weak explosion at "late" times (about 1 second rather than tens of milliseconds) in numerical experiments. Bethe and Wilson (1985) developed a theory of the process which involves neutrino diffusion from the core heating the infalling mantle. Because of the large opacity, this process is slow, and produces weak explosions. Other numerical experiments (Arnett. see below) do not confirm even weakly explosive behavior.

The bottleneck is the slow diffusion rate. This occurs in a region which has high entropy (due to strong shock heating). It is below a region of lower entropy (due to the weaker heating of the dying shock). This region of the mantle is hydrodynamically unstable to overturn. which transports energy and leptons to lower densities where diffusion can take over. This idea is distinct from previous discussion of overturn which involved the collapsed core (which is stable). While the theory is clear enough, numerical experiments run to such late times raise questions about the validity of the techniques used.

\section{VALIDITY OF NUMERICAL EXPERIMENTS}

\section{1. Correctness}

A fundamental problem for the scientist using a computer is that of validation of results. How do we know they are correct? An obvious and useless test is that of prejudice: the result seems "reasonable". While "unreasonable" results may be a useful clue for finding errors, the fact that results agree with our preconceptions does not imply that they are correct.

Test problems are somewhat more persuasive. Even so, we do not do research on problems to which we know the answer, so test problems are only a partial aid. Analytic solutions are seldom available for interesting new problems. If they were we would not need the computer. Discretization errors may be tested by repeating a calculation with different sizes of zone and time step: this is difficult if the job to be tested already pushes the practical limits of computer and budget. For some problems there are integral constraints which may be monitored to test the quality of computation (for example. energy conserva- 
tion or entropy "conservation": lepton conservation). These are only necessary but not sufficient for correctness. I think the best approach is to treat computer results as numerical experiments, and use strategles analogous to those of laboratory experimenters to establish reliability. Correspondingly. publication of the results of at least the most important of these tests is necessary for establishing a numerical experiment as more than a scenario.

\section{2. Some Types of Errors}

In the literature dealing with gravitational collapse one can find examples of many types of errors: (1) choice of physics (omitting crucial physical processes. using the wrong theory or too crude a version of the right theory). (2) choice of algorithms (e.g. . putting in the desired answer by choice of method of calculation). (3) programming blunders ("the only correct code is an obsolete one": this is especially difficult for those who must compute on different machines or who do not exploit modern techniques for software developement), and (4) discretization (truncation, roundoff, level of convergence, etc.).

\section{BIRTH OF NEUTRON STARS}

\section{1. Error Estimates}

\section{1. 1. Energy}

The rest energy of a Chandrasekhar mass $\left(M \simeq 1.5 M_{\Theta}\right)$ is $3 \cdot 10^{54}$ erg: the gravitational binding energy $B$ of a neutron star is about 0.1 of this. A weak explosion involves as little as $10^{50}$ ergs (e.g. . Crab Nebula?). Which is only $3 \cdot 10^{-4}$ of the binding energy released by core collapse. A typical computational sequence takes of order $10^{4}$ steps in time. Suppose we want an accuracy of $1 \%$ in explosion energy for this weak explosion. and that we make some small error in energy at each time step. If these errors are random (they grow as $\mathrm{Vn}$ ), then the fractional error per step is

$$
|\Delta E| / B \simeq 3 \cdot 10^{-8}
$$

For systematic errors (they grow as $n$ ). we have

$$
|\Delta E| / B \simeq 3 \cdot 10^{-10}
$$

These goals are much more stringent than usually required in computational work.

\section{1.2. Rates}

At present. "late times" mean about 1 second. If the weak explosion mentioned above were driven by some form of energy transfer, then the energy transfer rate would be $L \simeq 10^{50}$ erg/s. Now. the average over 
the collapsed core of the neutrino energy emissivity need to do this is

$$
\left\langle\epsilon>\simeq L / M \simeq 0.7 \cdot 10^{17} \mathrm{erg} / \mathrm{g} \mathrm{s}\right. \text {. }
$$

In the center, the true emissivity is

$$
\epsilon \simeq 10^{27} \mathrm{erg} / \mathrm{g} \mathrm{s} \text {. }
$$

which is closely balanced by the neutrino absorption rate. Numerically speaking. to avoid errors of $1 \%$ in $\langle\epsilon\rangle$. we need 12 significant figures for $\epsilon$ to cancel the corresponding absorption.

These estimates give a sense of the nature of the problem. Consider that at a given time. the state of the star is represented by an abstract vector whose components represent temperature, composition. neutrino group energy density, velocity, etc. at each selected point in space (each "zone"). This state vector is subjected to an evolution operator which updates each element to its value at the next time step. It is believed (without much proof) that crude state vectors (e.g. a a dozen energy groups to represent the neutrino distribution) are adequate. The errors discussed above are related to the evolution operator. and the problem posed is this: can we show that the cumulative errors in this evolution operator are smaller than the effect we seek?

\subsection{Numerical Experiment: $Y_{e}$ fixed}

A simple limiting case for which errors can be acceptably small is a collapse during which $Y_{e}$. the electron abundance per nucleon. is held fixed. As no neutrinos are formed, there is no neutrino cooling. The lepton pressure is a bit larger if all leptons are electrons. A third flaw is that $Y_{e}$ is frozen somewhat arbitrarily at a point after the core collapse begins $\left(\rho_{c} \simeq 2 \cdot 10^{10} \mathrm{~g} / \mathrm{cc}\right)$. Such a model gives a strong bounce, with the shock propagating all the way to the surface of the 4 $M_{\odot}$ helium star. Using a mild modification of Van Riper method for general relativistic hydrodynamics. the energy check showed a cumulative error of less than $10^{49}$ ergs, about $0.1 \%$ of the explosion energy ( $3.10^{51}$ ergs). An implicit, second order hydrodynamic scheme was used. so that the calculation could be continued to 3 minutes, at which point the envelope density was $\rho \simeq 10^{-2} \mathrm{~g} / \mathrm{cc}$. the shock had reached the surface. the outflow velocities were above escape velocity, and a neutron star of $1.1 \mathrm{M}_{\odot}$ had formed. Dynamic rezoning was necessary to resolve (for the first time) the steep density gradient between the neutron star and the ejecta ( $\rho$ changes by a factor of $10^{12}$ in a tiny fraction of the mass). Some thermonuclear burning occurred during the explosion. with $0.2 \mathrm{M}_{\odot}$ of ${ }^{56} \mathrm{Ni}$ being formed. This object would SNI-like light curve. Fig. 1 shows snapshots of the density structure at several different times, beginning with a well-evolved shock and continuing until after the shock reaches the structure of the hellum star.

The experiment fails on two predictions: (1) the gravitational mass ( $10 \%$ reduction for gravitational binding) is $1.0 \mathrm{M}_{\odot}$. so that such an event could not explain the masses in the binary pulsar PSR $1913+16$ (which are 1.45 and $1.38 M_{\rho}$ ), and (2) about $0.3 M_{\infty}$ of 


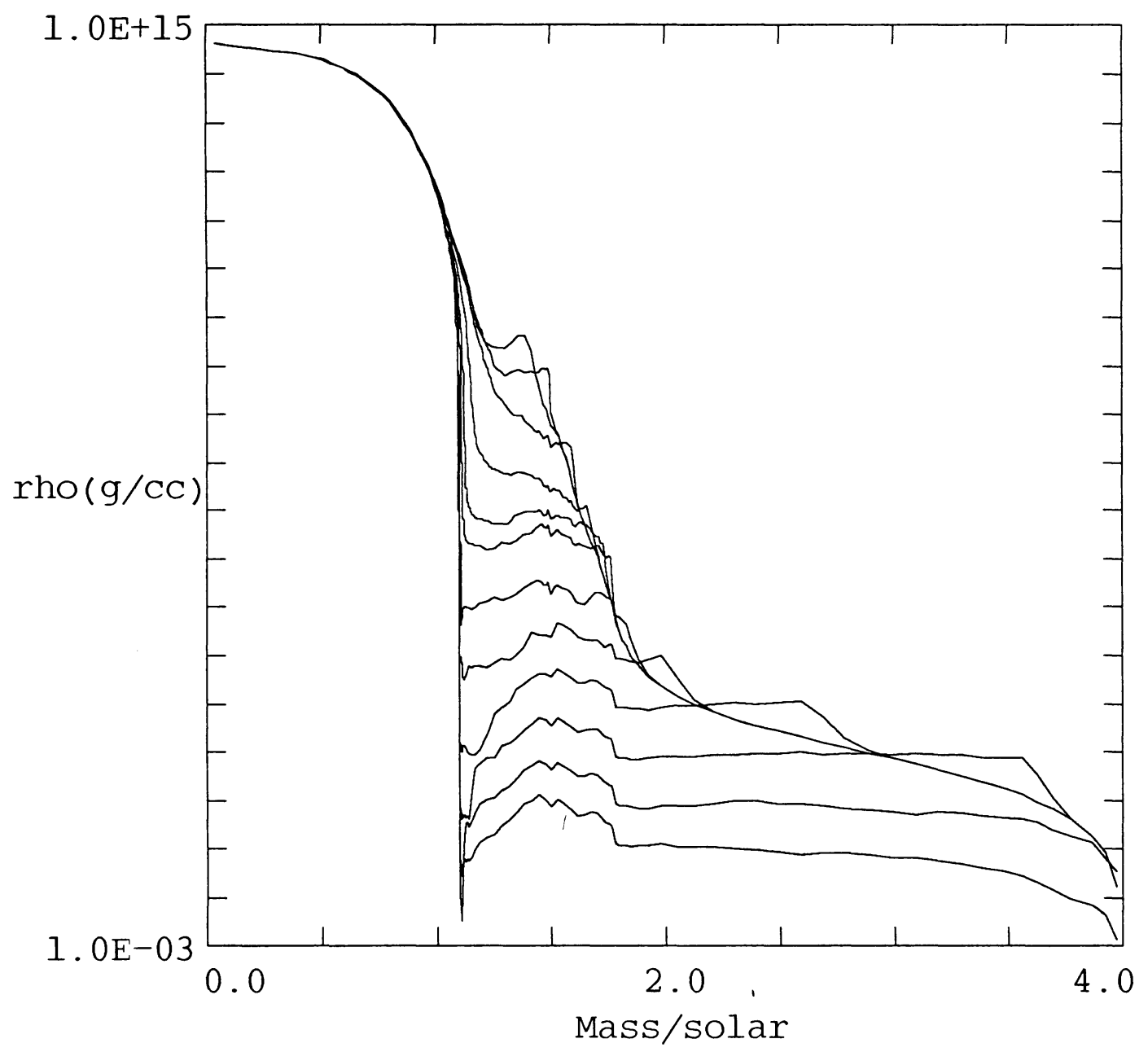

Figure 1. The formation of a neutron star. Density (in $\mathrm{g} \mathrm{cm}^{-3}$ ) is plotted versus Lagrangian mass coordinate (in solar masses): snapshots at several different times are shown. In the first snapshot the shock has propagated down to a density of about $10^{8} \mathrm{~g} \mathrm{~cm}^{-3}$. prior to the oxygen burning shell. Some explosive burning occurs: see the region from mass 1.4 to 1.8 solar units. Recombination of dissociated nuclel occurs in the mass above 1.1 solar units, and affects the hydrodynamics. Resolution of the steep density gradient at a mass of 1.1 requires special techniques: an implicit hydrodynamic scheme (second order. Lagrangian) with dynamic rezoning was used. The masses shown represent nucleon number, a conserved quantity: the gravitational mass of the neutron star is about 10\% less. 
${ }^{54} \mathrm{Fe}$ are ejected. This matter is already neutron rich when the collapse begins. is dissociated to nucleons, and recombines to give the shock a significant kick at late times. As ${ }^{56} \mathrm{Fe}$ is the most abundant isotope observed. this high yield of the rarer ${ }^{54} \mathrm{Fe}$ is an embarrassment.

\subsection{Numerical Experiment: Death of the Shock}

We repeat the previous experiment with weak interactions turned on. The core bounce is now weaker. but a strong shock begins to travel out to lower densities. It is weakened by nuclear dissociation. but is killed at low densities ( $\rho \simeq 10^{10} \mathrm{~g} / \mathrm{cc}$ ) by neutrino transport. As mentioned above. the mantle develops an unstable gradient, but if the code is not told about two dimensional phenomena. It happily ignores them. The calculation was continued to 1 second. with no hint of a late mechanism of the Wilson type. No further continuation was made because the energy check grew to be of the same order as the energy of Wilson's explosions (several times $10^{50}$ ergs).

\section{4. Advection and Explosion}

A direct integration of the buoyancy force gives turnover velocities of the order of $20 \%$ of sound speed. Which is enough to mix the mantle in 0.1 seconds. It appears that the crucial hydrodynamics is simply a large scale overturn rather than the more complex development of a turbulent cascade. It is easy to get explosions of the sort 1 found a long time ago. but hard to do the neutrino transport accurately in both the thick core and tenuous (spherical) mantle. That will be necessary to predict accurately the neutron star mass, and the energy and compositon of the ejecta.

Although we started with a spherical system. we were lead to a discussion of significant nonspherical motion. It is unlikely that an asymptotic calculation will lead us back to symmetry. Whether such phenomena can give new pulsars a kick of the magnitude suggested by observation remains to be seen.

\section{PROSPECTS}

The frontier of collapse research has now progressed to times of order seconds to minutes after the bounce. Which is considerably later than the 20 milliseconds discussed a few years ago. There are a number of prospective developments.

Radiation hydrodynamics of neutrinos at low depths is crucial for the late mechanisms, and for the possible survival of the shock with early mechanisms. Fu and Arnett (in preparation) have developed a method which should be an improvement over the flux-limiting technique now in use. with no computational penalty. Also. greater care in the consistent treatment of neutrino-matter coupling at high and low density will help with the energy check problem.

With the new hydrodynamic methods of Woodward and Colella, and van Leer. two and three dimension computations become feasible: the 
discussion above suggests that they are necessary. The question of a pulsar kick at birth is of prime importance: numerical experiments can be brought to bear on some mechanisms.

Observed neutron star masses (especially PSR 1913+16) have direct overlap with the results of late-time collapse experiments. Similarly. estimates of the nucleosynthesis yields are better if the ejection process actually falls out of the experiment. We may be approaching the point of seriously connecting young supernova remnants with evolutionary histories of their progenitor stars. Remnant energies. masses and compositions, as well as light curve and spectral data, are providing vital empirical information to which we can compare numerical experiment.

\section{REFERENCES}

Arnett, W. D. 1966 Can. J. Phys. 44. 2553.

Arnett. W.D. 1967 Can. J. Phys, 45. 1621.

Arnett. W. D. 1977a Ap. J. 218. 815.

Arnett, W. D. 1977 b Ap. J. Suppl. 35. 145.

Arnett. W. D. 1983 Ap. J. (Letters) 263. L55.

Bethe, H.A. Brown. G.E. Applegate, J. , and Lattimer. J.M. 1979 Nucl. Phys. A324. 487.

Bethe, H.A. and Wilson, J.R. 1985 Ap. J. 295. 14.

Colgate. S.A. and White, R. 1966 Ap. J. 143. 626.

Fuller. G. M. . Fowler. W. A. and Newman. M.J. 1982 Ap. J. 252. 715.

Hillebrandt. W. 1982 in Supernovae: A Survey of Current Research. ed. M.J. Rees and R.J. Stoneham (D. Reidel: Dordrecht).

Lattimer. J.M. 1981 Ann. Rev. Nucl. Particle Scl. . 31. 537.

Lattimer. J.M. and Burrows. A. 1984 in Problems of Collapse and Numerical Relativity. ed. D. Banal and $M$. Signore (D. Reldel: Dordrecht).

Mazurek. T. $1975 \mathrm{Ap}$. and Space Scl. 35. 117.

Nomoto. K. 1982 in Supernovae: A Survey of Current Research. ed. M.J. Rees and R.J. Stoneham (D. Reidel: Dordrecht).

Sato. K. 1975 Prog. Theor. Phys. 53. 595.

Weaver. T.A. Zimmerman. G.B. and Woosley. S.A. 1978 AR. J. 225. 1021.

Wilson. J.R. 1985 in Numerical Astrophysics, ed. J.M. Centrella. J.M. LeBlanc. and R. L. Bowers (Jones and Bartlett: Boston).

\section{DISCUSSION}

A. Burrows: You don't reproduce Wilson's results. How do your effective neutrinospheric temperature and neutrino luminosities compare with his?

W. Arnett: As you would guess, my temperatures and luminosities are less than Wilson's. The problem is not that the numerical experiments produce results which are obviously unphysical, but that they produce different, seemingly self-consistent results. 
T. Weaver: With regard to numerical errors, do you get convergence results when you reduce zone size, time steps, convergence criteria, etc.?

W. Arnett: It is difficult to summarize briefly two years of an extensive variety of collapse calculations, but basically the answer is yes, I do get convergent results. The errors which I worry most about are more subtle; over long times small effects can build, giving systematic differences. To the present level of zone size and time step, the calculations seem to converge; the most "accurate" ones do not explode by the Wilson mechanism.

S. Colgate: The large neutrino crossection due to neutral currents led to trapping during collapse of electron-capture neutrinos. The lepton-trapped neutron star core has only $\sim 1 / 10$ the binding energy of the lepton-depleted core. If neutrino oscillations $v_{c}$ - $\nu_{t}$ similar to but with a larger $\Delta \mathrm{m}$ than the $\nu_{c} \rightarrow \nu_{u}$ applied to the solar neutrino problem by Bethe occurred, we would have a lepton-free core, a strong accretion shock, and neutrino emission for neutrino ejection as in Colgate and White.

W. Arnett: These collapsing cores may be the only place in the Universe where the assumption of lepton conservation is of crucial importance. My strategy (which I have often questioned) is to get the standard case sorted out before exploring more exotic ones. 